\title{
Impact of COVID-19 Pneumonia on Neonatal Birth Outcomes
}

\author{
Nawsherwan ${ }^{1}$ - Suliman $\mathrm{Khan}^{2} \cdot{\text { Ghulam } \mathrm{Nabi}^{3} \cdot \text { Cuifang Fan }}^{4} \cdot$ Suqing Wang ${ }^{1}$
}

Received: 18 May 2020 / Accepted: 29 May 2020 / Published online: 22 June 2020

(C) Dr. K C Chaudhuri Foundation 2020

To the Editor: The coronavirus disease-2019 (COVID-19), a highly infectious disease has been declared as a pandemic by the World Health Organization (WHO) on 11th March 2020 [1]. Coronavirus such as severe acute respiratory syndrome coronavirus (SARS CoV), and Middle East respiratory syndrome coronavirus (MERS $\mathrm{CoV}$ ) has caused significant rates of adverse neonatal birth outcomes including neonatal mortality, stillbirth, low birth weight, and preterm delivery in pregnant women $[2,3]$. We aimed to investigate the possible impact of COVID-19 pneumonia on neonatal birth outcomes born to mothers with laboratory-confirmed COVID-19 pneumonia at Hubei Provincial Hospitals, China.

We conducted a case study of pregnant women $(n=7)$ infected with COVID-19 and admitted to five different Hubei Provincial Hospitals [Renmin Hospital of Wuhan University $(n=2)$, Huangshi Maternal and Child Health Hospital $(n=2)$, Jingzhou Maternal and Child Health Hospital $(n=1)$, Jingmen First Renmin Hospital $(n=1)$,

Nawsherwan and Suliman Khan contributed equally to this work.

Electronic supplementary material The online version of this article (https://doi.org/10.1007/s12098-020-03372-2) contains supplementary material, which is available to authorized users.

Cuifang Fan

359568292@qq.com

Suqing Wang

swang2099@whu.edu.cn

1 Department of Preventive Medicine, School of Health Sciences, Wuhan University, Wuhan 430071, Hubei, China

2 Department of Cerebrovascular Diseases, The Second Affiliated Hospital of Zhengzhou, Zhengzhou University, Zhengzhou, China

3 Key Laboratory of Animal Physiology, Biochemistry and Molecular Biology of Hebei Province, College of Life Sciences, Hebei Normal University, Shijiazhuang 050024, China

4 Department of Obstetrics and Gynecology, Renmin Hospital, Wuhan University, Wuhan 430060, Hubei, China
Zaoyang First Renmin Hospital $(n=1)]$ from Jan 29 through Feb 14, 2020. The COVID-19 pneumonia was diagnosed according to the New Coronavirus Pneumonia Prevention and Control Program (7th edition) [4]. Quantitative RT-PCR (qRTPCR) test for COVID-19 was conducted using specimens from the respiratory tract (nasopharyngeal swabs) and blood. The study protocol was approved by the Ethical Review Board of Hubei Provincial Central Hospital (Renmin Hospital of Wuhan University).

The pregnant women (27-34 y old) with COVID-19 pneumonia included in this study, delivered babies via $\mathrm{C}$-section in the third trimester of pregnancy. Two of seven pregnant women $(28.5 \%)$ had underlying medical conditions such as moderate anemia (patient $1 \& 5$ ). The laboratory results showed that three of seven $(42.8 \%)$ pregnant women had elevated concentrations of Creactive protein $(>10 \mathrm{mg} / \mathrm{L})$ and one pregnant woman $(14.2 \%)$ had lymphopenia $<1.1 \times 10^{9} / \mathrm{L}$. None of the pregnant women died. One of seven neonates $(14.2 \%$, neonate 5) had a low birth weight $(<2500 \mathrm{~g})$ and fetal distress (neonate 1$)$. Three of seven neonates $(42.8 \%)$ were delivered prematurely (neonates 3, 4 \& 5) and had Newborn Respiratory Distress Syndrome (NRDS) (neonates 3, 5 \& 7). Two of seven neonates $(28.5 \%)$ were transferred to a neonatal intensive care unit (NICU) and given noninvasive ventilation (neonates $4 \& 5$ ). All neonates were found negative for COVID-19 pneumonia (Supplementary Tables 1 and 2).

It is speculated that COVID-19 pneumonia in pregnant women may cause hypoxemia and increase the risk of adverse neonatal birth events including birth asphyxia and premature birth. Moreover, premature birth may be caused by maternal anemia in patient 5 . However, the direct relationship of COVID-19 with fetal distress still remaining elusive [5]. Based on our findings, development of COVID-19 pneumonia in pregnant women in the third trimester may be associated with adverse neonatal birth outcomes. A recently published report on ten neonates born to mothers with COVID-19 
pneumonia investigated that COVID-19 might have an adverse effect on neonatal birth outcomes [6]. In addition, SARS CoV and MERS CoV were associated with adverse neonatal birth outcomes in pregnant women [2, 3].

The symptoms manifested by patients with COVID19 infection were similar to those of patients reported in previously published studies [7]. Three of seven patients had higher level of C-reactive protein $(>10 \mathrm{mg} / \mathrm{L})$, as observed in a previous study [7]. All seven patients underwent $\mathrm{C}$-section. In one of the seven patients, the indication of $\mathrm{C}$-section was fetal distress. Moreover, the risk of COVID-19 transmission from mother to the neonate during intrapartum by vaginal delivery was another reason for conducting $\mathrm{C}$-sections. This study had a small sample size and some of the data regarding neonatal birth outcomes were missing.

In summary, COVID-19 pneumonia in pregnant women may have an adverse effect on neonatal birth outcomes, causing problems including premature birth, fetal distress, and NRDS.

Acknowledgements We are thankful to the staff of Obstetrics and Gynecology, Department of Renmin Hospital, Wuhan for helping in data collection.

\section{Compliance with Ethical Standards}

Conflict of Interest None.

\section{References}

1. World Health Organization. WHO Director-General's Opening Remarks at the Media Briefing on COVID-19. Available at: https:// www.who.int/dg/speeches/detail/who-director-general-s-openingremarks-at-the-media-briefing-on-covid-19\%2D\%2D-11-march2020. Accessed March 11, 2020.

2. Wong SF, Chow KM, Leung TN, et al. Pregnancy and perinatal outcomes of women with severe acute respiratory syndrome. Am J Obstet Gynecol. 2004;191:292-7.

3. Alfaraj SH, Al-Tawfiq JA, Memish ZA. Middle East respiratory syndrome coronavirus (MERS-CoV) infection during pregnancy: Report of two cases \& review of the literature. J Microbiol Immunol Infect. 2019;52:501-3.

4. National Health Commission of People's Republique of China: Diagnosis, Treatment and Prevention Program of Coronavirus Disease (COVID-19) (Pilot edition 7). Available at: http://www.nhc. gov.cn/yzygj/s7653p/202003/46c9294a7dfe4cef80dc7f5912eb1989/ files/ce3e6945832a438eaae415350a8ce964.pdf. Accessed 21 March 2020.

5. Working Group for the Prevention and Control of Neonatal 2019-nCoV Infection in the Perinatal Period of the Editorial Committee of Chinese Journal of Contemporary Pediatrics. Perinatal and neonatal management plan for prevention and control of 2019 novel coronavirus infection (1st Edition). Chin J Contemp Pediatr. 2020;22:87-90.

6. Zhu H, Wang L, Fang C, et al. Clinical analysis of 10 neonates born to mothers with 2019-nCoV pneumonia. Transl Pediatr. 2020; Doi.org/10.21037/tp.2020.02.06.

7. Chen H, Guo J, Wang C, et el. Clinical characteristics and intrauterine vertical transmission potential of COVID-19 infection in nine pregnant women: A retrospective review of medical records. Lancet. 2020; https://doi.org/10.1016/S0140-6736(20)30360-3.

Publisher's Note Springer Nature remains neutral with regard to jurisdictional claims in published maps and institutional affiliations. 